\title{
AUTORREGULAÇÃO EMOCIONAL NA PERSPECTIVA SOCIAL COGNITIVA: UMA REVISÃO INTEGRATIVA
}

\author{
EMOTIONAL SELF-REGULATION IN THE COGNITIVE SOCIAL \\ PERSPECTIVE: AN INTEGRATIVE REVIEW \\ AUTORREGULACIÓN EMOCIONAL DESDE LA PERSPECTIVA
SOCIAL COGNITIVA: UNA REVISIÓN INTEGRATIVA
}

\author{
Jamille Gabriela CunHa da Silva ${ }^{\mathrm{I}}$ \\ LUCIANA AMARAL GaRCia ${ }^{\mathrm{I}}$ \\ MaÉly Ferreira Holanda Ramos ${ }^{\mathrm{I}}$ \\ IUniversidade Federal do Pará (UFPA), Belém/PA - Brasil
}

Resumo O homem, segundo a Teoria Social Cognitiva, é capaz de autorregular seu comportamento, motivações, emoções, dentre outros aspectos. Esta pesquisa tem por objetivo construir um panorama das pesquisas nacionais e internacionais sobre autorregulação emocional na perspectiva social cognitiva, por meio de uma revisão integrativa da literatura, considerando o período de 2008 a 2019; identificando o que os autores têm abordado sobre esta temática e apontando possíveis lacunas. Com uma abordagem quantitativa, o referido estudo apropriou-se de uma análise de frequência mediante a utilização do software NodeXL, que gerou um Grafo com dados de medidas de centralidades adotadas a fim de destacar os construtos mais relevantes. Os resultados apontaram para cinco artigos analisados que apresentaram a relevância de quatro construtos: Emotional intelligence; Emotional competence; Emotion regulation; Coping. Os dados demonstram que a literatura tem afirmado que o sujeito que é capaz de autorregular-se emocionalmente apresenta uma inteligência emocional satisfatória, além de torna-se competente para enfrentar situações que podem gerar um estado emocional negativo. Além disso, faz-se necessário investir em programas que estimulem a competência emocional nos indivíduos a fim de garantir a eficácia no gerenciamento das emoções.

Palavras-Chave: Autorregulação emocional; Emoções; Alunos; Teoria social COGNITIVA.

AbSTract Man is constituted as an autonomous subject who, according to the Cognitive Social Theory, is able to self-regulate his behavior, motivations, emotions, among other aspects. This research aims to build an overview of national and international research on emotional 
self-regulation in the cognitive social perspective, through an integrative literature review, considering the period from 2008 to 2019; identifying what the authors have approached about this theme and pointing out possible gaps. With a quantitative, this study appropriated a frequency analysis using the NodeXL software, which generated a graph with data of centrality measures adopted in order to highlight the most relevant constructs. The results pointed to five analyzed articles that presented the relevance of four constructs: Emotional intelligence; Emotional competence; Emotion regulation; Coping These data demonstrate that the literature has stated that the subject who is capable of self-regulating emotionally has satisfactory emotional intelligence, and is competent to face situations that can generate a negative emotional state. In addition, it is necessary to invest in programs that stimulate emotional competence in individuals to ensure effective management of emotions.

Keywords: Emotional self-regulation; Emotions; Students; Cognitive social THEORY.

Resumen Según la teoría cognitiva social, el hombre es capaz de autorregular su comportamiento, motivaciones, emociones, entre otros aspectos. Esta investigación tiene como objetivo construir una visión general de la investigación nacional e internacional sobre autorregulación emocional en la perspectiva cognitiva social, a través de una revisión de literatura integradora, considerando el período de 2008 a 2019; identificando lo que los autores han abordado sobre este tema y señalando posibles lagunas. Con un enfoque cuantitativo, el estudio referido se apropió de un análisis de frecuencia utilizando el software NodeXL, que generó un Gráfico con datos de medidas de centralidades adoptadas para resaltar las construcciones más relevantes. Los resultados apuntaban a cinco artículos analizados que presentaban la relevancia de cuatro constructos: inteligencia emocional; Competencia emocional; Regulación emocional; Albardilla. Los datos demuestran que la literatura ha declarado que el sujeto que es capaz de autorregularse emocionalmente tiene una inteligencia emocional satisfactoria, además de ser competente para enfrentar situaciones que pueden generar un estado emocional negativo. Además, es necesario invertir en programas que estimulen la competencia emocional en las personas para garantizar la efectividad en el manejo de las emociones.

Palabras Clave: Autorregulación emocional; Emociones; Estudiantes; Teoría soCIAL COGNITIVA.

\section{INTRODUÇ̃̃̃o}

$\mathrm{O}$ ato de estabelecer metas é recorrente no cotidiano de cada sujeito, visto que o desejo de alcançar objetivos é comum entre os indivíduos de uma sociedade ativa. Propor objetivos e criar meios para alcançá-los fazem parte de um construto amplo que é utilizado em diversas perspectivas, a saber: a regulação (BANDURA, 1991). Quando o sujeito regula seu próprio comportamento a fim de conquistar resultados positivos, este conceito passa a ser denominado como "autorregulação". Ou seja, regulação consiste no ato de controlar comportamentos por meio de estratégias que podem ser coletivas; entretanto, quando esse 
controle é efetuado por um indivíduo que busca gerenciar o seu próprio comportamento, este se denomina de "autorregulação". Ambos os processos, apesar dessa pequena distinção, fazem parte de uma consciência funcional que norteia os cursos de ação adotados pelo ser humano (BANDURA, 2005).

Algumas teorias que estudam o comportamento humano, utilizam-se do construto da autorregulação com o intuito de explicar condutas básicas do homem. Na perspectiva sociocognitivista, por exemplo, na qual este estudo está baseado, a autorregulação é utilizada como um mecanismo consciente, voluntário e interno do indivíduo que possibilita ao mesmo a capacidade de estabelecer o controle sobre suas ações, sentimentos e pensamentos com o intuito de alcançar resultados esperados. É por meio deste processo multifacetado que o sujeito frequentemente se automonitora, esforçando-se para alcançar os objetivos pré-estabelecidos, sem esquecer-se de julgar e avaliar seu desempenho neste processo (BANDURA; AZZI; POLYDORO, 2008, p. 151; BANDURA, 1991; SCHUNK, 2012; POLYDORO, 2017; p. 13).

A Teoria Social Cognitiva de Albert Bandura reconhece que o ser humano baseia grande parte das suas escolhas, e consequentemente seu comportamento, na intencionalidade que exerce a função de norteadora dos cursos de ação adotados. Nessa visão, o comportamento é constituído por uma estrutura causal que é conhecida como "causalidade recíproca" ou ainda "determinismo recíproco". De acordo com essa estrutura o comportamento humano é constituído por uma tríade composta por: (1) fatores pessoais - que consistem nas características cognitivas do sujeito, além de seus aspectos afetivos, biológicos e suas crenças pessoais; (2) fatores ambientais - nos quais envolvem o ambiente físico, social e estrutural, ou seja, o externo ao indivíduo; (3) comportamento - que consiste nos próprios cursos de ação do sujeito, sejam ações físicas, verbais ou tomadas de decisão (BANDURA, 1999; AZZI, 2014, p. 28).

Nesta perspectiva, os indivíduos são ser agêntico que apresentam a capacidade de proatividade, autorreflexão, pensamento antecipatório, além de poder mediar os efeitos diretos causados pelas influências externas que podem afetar sua crença individual (BANDURA, 1991; BANDURA, 2005). Na Teoria Social Cognitiva a agência humana possui quatro categorias básicas: (1) intencionalidade - refere-se a intenção de executar um comportamento futuro, comprometendo-se a realiza-lo; (2) antecipação - materializa-se no pensamento antecipatório projetando objetivos e possíveis resultados; (3) autorreatividade - consiste na prática do que fora projetado, regulando-se para alcançar os resultados esperados; (4) autorreflexão - que constitui-se na avaliação de todo o processo, e se necessário for, estabelece novos cursos de ação para conseguir êxito no que fora almejado (BANDURA; AZZI; POLYDORO, 2008).

O construto da agência humana está estritamente ligado a autorregulação, visto que é por meio desse primeiro processo que o sujeito estabelece objetivos nos quais ele deve regular seu próprio comportamento com vista ao alcance de resultados positivos em diversos segmentos. Dentro da autorregulação, existem alguns processos subcognitivos que direcionam o ser agêntico no seu processo autorregulatório: auto-observação, processos de julgamento e autorreações.

O subprocesso da auto-observação é fundamental para disponibilizar informações significativas que norteiam a definição dos objetivos, além de dar suporte na avaliação 
de todo o percurso de alcance desses objetivos (BANDURA; AZZI; POLYDORO, 2008; BANDURA, 1991). O segundo subprocesso conhecido como julgamento exerce a função de analisar as condutas adotadas, refletindo se elas são positivas ou negativas para o êxito nos resultados esperados. Para tanto, algumas dimensões são estabelecidas dentro desse subprocesso, a saber: referências sociais que estão contidas nas trocas de experiências ou referências pessoais que consistem nas experiências do próprio ser agêntico; o valor dado à referida atividade visto que demonstra o esforço a ser empenhado na mesma demonstra $\mathrm{o}$ quão significante é a progressão para o alcance dos resultados propostos; e o lugar no qual o comportamento é executado que demonstra certa influência na construção dos cursos de ação do indivíduo (BANDURA, 1991; BANDURA, 1999; BANDURA; AZZI; POLYDORO, 2008; AZZI, 2014).

O último subprocesso consiste na autorreação que é decorrente dos anteriores. A partir do julgamento são definidas as próximas ações do indivíduo, ou seja, se o parecer for favorável significa que as reações adotadas pelo mesmo foram satisfatórias e devem ser mantidas, caso contrário se o julgamento for negativo o sujeito precisa alterar seus cursos de ação adotados. Dentro deste subprocesso, existem três tipos de autorreação: a avaliativa - nessa o sujeito avalia a partir dos padrões de referências pessoais ou sociais supracitados que a partir dos resultados obtidos podem promover motivação na regulação dos atos; tangível - nesse tipo de autorreação existe a possibilidade de reforçar o comportamento adotado ou a punição pelo mesmo decorrente das consequências das escolhas do sujeito; inexistentes - nessa, como o próprio nome sugere, o indivíduo não efetua nenhum tipo de conduta estabelecendo um estado de inércia (BANDURA; AZZI; POLYDORO, 2008; BANDURA, 1986).

Como fora mencionado, o construto da autorregulação é amplamente aplicável e existem estudos que o relacionam com vários aspectos do comportamento humano, inclusive com fatores emocionais como é caso da autorregulação emocional. Também chamada de autorregulação do afeto, esta categoria aborda a possibilidade de o sujeito identificar e gerenciar suas emoções, além de identificar as prováveis consequências de cada emoção adotada (BANDURA; AZZI; POLYDORO, 2008; BANDURA; CAPRARA; BARBARANELLI, 2003).

A autorregulação emocional, na qual consiste no foco do presente estudo, está relacionada a processos de gerenciamento e controle de aspectos fisiológicos e expressivos da emoção que podem proporcionar ao sujeito a capacidade de regular suas próprias expressões emocionais. As emoções são inatas ao ser humano - sejam elas de natureza positiva como a alegria, ou negativa a exemplo a tristeza - e a necessidade de um autogerenciamento periódico dessas emoções faz-se necessário visto que, na ausência do mesmo, o bem estar físico e psíquico do ser humano pode ficar comprometido (GROSS, 1999; 2002; DAMÁSIO, 1996).

Também em uma perspectiva cognitivista, Garber, Walker e Zeman (1991) dentro da Teoria do Processamento da Informação, construiu um modelo visando o aperfeiçoamento da autorregulação emocional, corroborando com aspectos afetivos possibilitando um controle mais efetivo das emoções. O referido modelo é constituído por seis etapas: (1) percepção da emoção apresentada e, se for necessário, o controle da mesma; (2) identifi- 
cação do que motivou esta emoção e do que poderia ter sido feito para evitar a mesma; (3) estabelecimento de metas a fim de gerenciar o afeto apresentado; (4) identificar possíveis respostas que podem ser alcançadas mediante do comportamento adotado; (5) avaliação deste processo; (6) avaliação do desempenho pessoal quanto ao que fora planejado.

Mediante o modelo apresentado, constituíram-se algumas estratégias que ajudam na autorregulação emocional do sujeito, essas estratégias de enfrentamento podem ser utilizadas de maneira consciente ou inconsciente e proporcionam amparo para modificação ou manutenção dos estados emocionais (GROSS, 1998; BORTOLETTO; BORUCHOVITCH, 2013). Vale ressaltar que elas podem ser desempenhadas de forma isolada, mas também de forma a relacionarem-se entre si (BORTOLETTO; BORUCHOVITCH, 2013).

Existe uma gama de estratégias de enfrentamento emocional, entretanto, segundo os autores Garnefski, Kraaij e Spinhoven (2001) as mais utilizadas são: aceitação da situação e emoções vividas; supressão da expressão, ou seja, uma tentativa de diminuir as expressões emocionais; disfarce como forma de camuflar a emoção presente; externalização de sentimentos via ações verbais e/ou físicas; reavaliação positiva da situação a fim de extrair lições de aprendizado do ocorrido; autoculpabilização que refere-se a atribuição de culpa exclusivamente para si quanto ao estado emocional vivido; ruminação, ou seja, o pensamento exagerado sobre o estado afetivo que o mesmo se encontra; afastamento do referido evento; busca pelo apoio social com entes queridos; distração através do envolvimento com tarefas diferenciadas; etc.

A aplicação dessas estratégias visando a autorregulação das emoções apresentadas, tem a capacidade de influenciar diretamente o comportamento humano, a motivação, além do desempenho profissional e acadêmico. Realizar um gerenciamento emocional eficaz pode evitar o surgimento de doenças psicossomáticas, como depressão e crises de ansiedade, podendo causar o comprometimento no desempenho social e no processo de aprendizagem (CAPRARA et al., 2013).

Estudos indicam que a falta de gerenciamento emocional pode provocar esgotamento físico, emocional e mental no indivíduo afetando diretamente no desenvolvimento de atividades acadêmicas, na socialização e na organização (FRIED, 2010; 2011; SANTOS; PRIMI, 2014). Para evitar o adoecimento do indivíduo, faz-se necessário investir na autorregulação das emoções com vista ao alcance do equilíbrio e bem-estar emocional (CARLOTTO, 2002).

A ausência desse gerenciamento tem afetado o desempenho acadêmico de estudantes em todas as modalidades de ensino, seja na educação básica ou na universidade. Na pós-graduação, por exemplo, muitos alunos precisam administrar responsabilidades no lar, no emprego, no curso de mestrado e doutorado, dentre outras. Essa gama de responsabilidade exige um maior domínio sobre as emoções, pois do contrário, esses alunos terão maiores dificuldades e prejuízos em suas relações sociais e no seu processo de aprendizagem. Além disso, a perda de interesse com tamanhas dificuldades, pode acometer muitos pós-graduandos (BRANDTNER; BARDAGI, 2009).

Segundo Storrie, Ahern e Tuckett (2010), os maiores problemas encontrados nas universidades estão relacionados à ansiedade, sintomas de estresse, depressão que acarretam o insucesso acadêmico. A partir deste contexto faz-se necessário investigar mais sobre a influência da autorregulação no cotidiano de alunos da pós-graduação. Apesar da gama de 
estudos relacionados aos aspectos socioemocionais, ainda existem lacunas relacionando o conceito de autorregulação emocional com o desempenho de pós-graduandos (PEÑA-SARRIONANDIA; MIKOLAJCZAK; GROSS, 2015). Principalmente no que tange a perspectiva da Teoria Social Cognitiva, que consiste em uma teoria relativamente nova, mas que apresenta construtos bem estruturados.

Mediante ao exposto, a presente pesquisa tem como objetivo principal construir um panorama das pesquisas nacionais e internacionais sobre autorregulação emocional na perspectiva social cognitiva em alunos da pós-graduação por meio de uma revisão integrativa da literatura, delimitando-se ao período de 2008 a 2019. A referida pesquisa buscou identificar estudos referentes ao construto da autorregulação emocional - considerando também o termo "regulação emocional" - a fim de perceber o que os autores têm produzido acerca da temática e contribuir com a atualização das discussões sobre o conceito eliminando possíveis lacunas encontradas por outros estudos.

\section{Percurso metodológico}

A presente pesquisa caracteriza-se como uma revisão integrativa da literatura na qual consiste em um tipo de abordagem que consegue tratar de um fenômeno relevante possibilitando a utilização de estudos com diversos métodos, seja ele experimental ou não (WHITTEMORE; KNAF, 2005). Para tal, foram estabelecidas sete etapas para a realização da referida revisão, a saber: a) formulação da pergunta problema; b) localização e seleção dos estudos; c) avaliação crítica dos estudos; d) coleta dos dados; e) análise e apresentação dos dados; f) interpretação dos dados; (g) aprimoramento e atualização da revisão (RAMOS, 2015).

A primeira etapa da pesquisa (etapa A) constituiu-se pela construção da pergunta problema utilizando-se da técnica "PVO" - onde "P" significa participantes, contexto ou ainda situação problema; "V" consiste na variável de estudo; "O" significa os resultados esperados (RAMOS, 2015). Portanto, no presente estudo: $\mathrm{P}$ - significou os alunos de pós-graduação; V - deu-se mediante o construto da autorregulação emocional, mas também foi aceito o termo regulação emocional; $\mathrm{O}$ - foi a construção do próprio panorama dos estudos sobre o tema sugerido. Este processo resultou na seguinte questão: "Qual o panorama das pesquisas sobre a autorregulação emocional em alunos de pós-graduação considerando o período de 2008 a 2019?”. Ressalta-se que após as buscas não foram encontrados estudos aplicados ao contexto da pós-graduação, sendo assim redimensionou-se a pergunta excluído o P (alunos de pós-graduação), resultando no seguinte problema: "Qual o panorama das pesquisas sobre a autorregulação emocional no período de 2008 a 2019?".

Esse processo foi necessário para que a pesquisa pudesse ser desenvolvida de maneira efetiva. Além disso, faz-se necessário repensar sobre a necessidade de pesquisas que busquem relacionar os conceitos de autorregulação emocional e a pós-graduação, visto a importância do gerenciamento emocional nesta etapa de ensino, devido a gama de responsabilidades e compromissos, como já fora mencionado.

$\mathrm{Na}$ etapa B - localização e seleção dos estudos - caracterizou-se pela definição dos descritores, a saber: Autorregulação emocional; regulação emocional; pós-graduação. Além disso, foram definidas as plataformas digitais nas quais estas buscas deveriam ser 
realizadas: Periódicos da Capes - plataforma com uma biblioteca virtual de referência nacional e internacional; Scielo - periódico de grande reconhecimento no meio acadêmico; Lilacs - conhecido por divulgar amplos estudos na América Latina; ERIC - periódico com biblioteca exclusivamente na língua inglesa, entretanto reconhecido internacionalmente pelos estudos disponibilizados.

$\mathrm{Na}$ terceira etapa - etapa $\mathrm{C}$ - foram construídos alguns critérios de inclusão para a avaliação crítica e seleção dos estudos, foram eles: (1) publicados nos últimos 10 anos; (2) Somente artigos científicos; (3) artigos em português ou em inglês ou em espanhol; (4) com descritores no título ou palavras-chave; (5) disponível gratuitamente; (6) artigos na íntegra; (7) revisado por pares; (8) a luz da Teoria Social Cognitiva - esta teoria foi adotada afim de destacar a importância de estudos que considerem aspectos sociais e cognitivos, além de colaborar com a ampliação e fomento das discussões relacionando-a ao construto da autorregulação emocional. Os estudos que não atenderam a todos os critérios estabelecidos, foram descartados.

A quarta etapa (etapa D) caracterizou-se pelo processo de coleta de dados que se deu pela identificação das seguintes variáveis: (1) Ano de publicação; (2) Metodologia adotada; (3) Qualis das revistas em que os estudos foram publicados; (4) Palavras-chave. Na etapa E, para a análise e apresentação dos dados, foi utilizado uma abordagem quantitativa, na qual utilizou-se uma análise de frequência e para corroborar com os dados obtidos, aplicou-se a análise de dados através do software NodeXL gerando Grafos e medidas de centralidade.

A etapa $\mathrm{F}$ constituiu-se pela interpretação dos resultados obtidos que se deu por meio da Teoria Social Cognitiva de Albert Bandura (1986). Para finalizar a pesquisa, a etapa G se referiu ao aprimoramento do texto final da revisão sistemática concluindo o estudo proposto.

\section{RESULTADOS E DISCUSSÃo}

\section{Processo de refinamento do material selecionado}

Para realizar o levantamento inicial foram utilizados alguns cruzamentos com os descritores principais, a saber: "Autorregulação emocional AND pós-graduação"; "Emotional self-regulation AND postgraduate"; "Autorregulação emocional"; "Emotional self-regulation; "Regulação emocional AND pós-graduação"; "Emotional regulation AND postgraduate"; "Regulação emocional"; "Emotional Regulation". Vale ressaltar que os artigos deveriam apresentar esses descritores no título, resumo ou em suas palavras-chave.

O resultado das buscas, após a aplicação de todos os critérios, indicou a ausência de artigos aplicados à pós-graduação, interesse primário deste estudo. Tal dado evidencia a importante lacuna que se tem neste campo de pesquisa quando se trata de autorregulação emocional, conceito situado na Teoria Social Cognitiva (TSC). Sabe-se que os estudos com TCS no Brasil ainda estão em crescimento e, portanto, determinados construtos aplicados a contextos específicos ainda são insipientes. Este resultado apenas ressalta a necessidade de se ampliar o número de pesquisas sobre a autorregulação emocional na Pós-graduação, levando em consideração que este contexto é vulnerável a instabilidades emocionais, estresse, adoecimento mental, dentre outros aspectos que podem resultar em doenças psicos- 
somáticas despertando um sentimento de desinteresse no prosseguimento desse processo formativo (BRANER; BARDAGI, 2009; BATISTA et al., 2016).

Após a realização desse levantamento inicial e a ausência de materiais que contemplassem todos os critérios pré-estabelecidos, decidiu-se refazer as buscas utilizando apenas os descritores "autorregulação emocional" e "regulação emocional", ampliando as possibilidades de investigação do construto. Nesta nova busca, na base de dados CAPES periódicos foram encontrados inicialmente 98.358 que após a aplicação de todos os critérios de inclusão resultaram em 5 artigos. Nas bases Scielo e Lilacs não foram encontrados resultados dos cruzamentos citados. Enquanto na biblioteca ERIC foi levantado inicialmente 359.399 arquivos, porém, após a aplicação dos critérios todos os arquivos foram descartados. A Tabela 1 apresenta o total de artigos levantados após a aplicação dos critérios de inclusão e a exclusão de materiais repetidos.

Tabela 1 - Total de artigos sobre a autorregulação emocional na perspectiva social cognitiva, levantados nos sítios da CAPES, Scielo, Lilacs e ERIC, no período de 2009 a 2019.

\begin{tabular}{lrrr}
\multicolumn{1}{c}{ Diretórios } & $\begin{array}{c}\text { Total de artigos } \\
\text { levantados }\end{array}$ & $\begin{array}{c}\text { Total após a aplicação } \\
\text { dos critérios de inclusão }\end{array}$ & $\begin{array}{c}\text { Total excluindo } \\
\text { os repetidos }\end{array}$ \\
\hline Capes & 98.358 & 8 & 5 \\
Scielo & 0 & 0 & 0 \\
\hline Lilacs & 0 & 0 & 0 \\
ERIC & 359.399 & 0 & 0 \\
\hline TOTAL & $\mathbf{4 5 7 . 7 5 7}$ & $\mathbf{8}$ & $\mathbf{5}$ \\
\hline
\end{tabular}

Fonte: Elaborado pelas Autoras (2019).

No total, 457,765 objetos foram excluídos por não contemplarem os critérios pré-estabelecidos. Deste montante, cerca de $74 \%$ foram excluídos por não estarem de acordo com os critérios 1 e 4 - (1) publicados nos últimos 10 anos; (4) com descritores no título ou palavras-chave. O restante, $26 \%$, foi descartado por não se tratar de artigos científicos - correspondendo ao critério 2 -, ou seja, a maior parte dos objetos levantados foram excluídos nos primeiros critérios estabelecidos. Após a aplicação dos critérios de inclusão, apenas 5 artigos foram selecionados para análise. Esta análise foi organizada em dois segmentos que serão abordados nos próximos subtópicos, a saber: (1) Caracterização dos estudos selecionados; (2) Rede semântica com as palavras-chave.

\section{Caracterização do material COLHIdo}

\section{Ano de publicação}

Os estudos selecionados foram publicados recentemente, a considerar o recorte temporal estabelecido no primeiro critério de inclusão - os artigos deveriam ter data de publicação nos últimos dez anos. Os estudos apresentados foram publicados em 2010 (1 artigo), 2013 (1 estudo), dois artigos tiveram publicação em 2015 e um foi publicado em 2017. Esses dados podem significar que o estudo sobre a temática em questão ainda é lacunar sendo 
necessário maior incremento, principalmente no que tange a pesquisas empíricas realizadas na América Latina, sobretudo no Brasil. Notou-se que mesmo ampliando o contexto os achados ainda são poucos. Na Tabela 2 é possível identificar os cinco estudos selecionados, com algumas informações básicas, a saber: título, autoria e ano de publicação.

Tabela 2 - Título, autoria e ano de publicação dos artigos sobre a autorregulação emocional na perspectiva social cognitiva, selecionados para análise em função do refinamento realizado, 2019.

\begin{tabular}{lrl}
\hline \multicolumn{1}{c}{ Título } & Ano & \multicolumn{1}{c}{ Autores } \\
\hline $\begin{array}{l}\text { Emotional Intelligence, Emotional Self-regulation and Dis- } \\
\text { positional Mindfulness in High School Gifted Students } \\
\begin{array}{l}\text { Learning Strategies and Emotional Regulation of Peda- } \\
\text { gogy Students }\end{array}\end{array}$ & $2017 \begin{array}{l}\text { Heshmati, R; Ahma- } \\
\text { dkhanloo, E. }\end{array}$ \\
\hline $\begin{array}{l}\text { Regulação Emocional: A Construção De Um Instrumento } \\
\text { E Resultados Iniciais }\end{array}$ & $2010 \begin{array}{l}\text { Bortoletto, D; } \\
\text { Boruchovitch, E. }\end{array}$ \\
$\begin{array}{l}\text { Parental self-regulation, emotional regulation and } \\
\text { Bemperament: Implications for intervention }\end{array}$ & $2015 \begin{array}{l}\text { Barros, L; } \\
\text { Goes, A. R; } \\
\text { Pereira, A. I. }\end{array}$ \\
\hline $\begin{array}{l}\text { Integrating emotion regulation and emotional intelligence } \\
\text { traditions: ameta-analysis }\end{array}$ & $2015 \begin{array}{l}\text { Peña-Sarrionandia A; } \\
\text { Mikolajczak, M; } \\
\text { Gross, J. J }\end{array}$ \\
\hline
\end{tabular}

Fonte: Elaborado pelas Autoras (2019).

\section{Metodologia dos eStudos}

As metodologias adotadas pelos estudos envolveram perspectivas teóricas e empíricas. Do total de cinco artigos, dois foram de natureza teórica, com isso faz-se necessário destacar que estudos teóricos são necessários para a construção de novas ideologias, ou aprimoramento de conceitos e teorias já preestabelecidas promovendo condições para possíveis intervenções na realidade (DEMO, 2000). Os três estudos restantes, apresentaram natureza empírica, dois consideraram o contexto escolar no Brasil e no Irã e o outro foi realizado no contexto acadêmico da graduação no Estado de Minas Gerais/Brasil. Destaca-se que estudos empíricos são de grande valia para a construção de novas percepções, possibilitando maior apropriação e concretude da realidade, visto que os pesquisadores foram diretamente no lócus da pesquisa analisar o construto explorado (DEMO, 1996).

Dos estudos teóricos, a pesquisa de Barros, Goes e Pereira (2015), que teve como objetivo compreender o que a literatura tem indicado sobre os processos da autorregulação e do ajustamento emocional dos pais na criação dos filhos e as principais estratégias dessa autorregulação; foi caracterizada como revisão integrativa que consiste em um método que sistematiza os estudos significativos sobre o tema utilizando-se de uma síntese dos mesmos (SOUZA; SILVA; CARVALHO, 2010). Neste estudo, as autoras levantaram artigos que abordassem temáticas acerca da parentalidade, os processos de autorregulação e regulação emocional dos mesmos e suas influências no desenvolvimento da criança. 
O outro estudo teórico consiste em uma revisão da literatura que se constitui através de materiais já publicados e disponíveis na internet, principalmente artigos científicos e livros (GIL, 1991). Nesta pesquisa, que teve como objetivo realizar uma revisão da literatura sobre inteligência emocional x regulação emocional, Peña-Sarrionandia, Mikolajczak e Gross (2015) levantaram estudos até outubro do ano de 2014 utilizando as seguintes bases de dados: Scopus; PsycINFO; Pubmed online. Os autores utilizaram o cruzamento dos seguintes construtos: Inteligência emocional, regulação de emoções, seleção de situações, modificação de situação, desenvolvimento de atenção, mudança cognitiva, modulação de resposta, prevenção, previsão, enfrentamento, resolução de conflitos, solução de problemas, apoio, distração, concentração, ruminação, mente plena e mente vagando, avaliação de ameaça, avaliação de desafio, autoeficácia, reavaliação, aceitação, reação de superaquecimento, supressão, uso de substâncias, autoagressão. Um total de 90 estudos foram analisados utilizando análise estatística de associação entre os construtos Inteligência Emocional a Regulação Emocional.

Os autores Heshmati e Ahmadkhanloo (2017) realizaram uma pesquisa com o intuito de estudar a relação entre inteligência emocional, "mindfulness" - em tradução livre: "atenção plena" ou "plena mentalidade" - e autorregulação emocional em estudantes superdotados que cursam o ensino médio. O estudo empírico de caráter quantitativo - no qual possibilita mensurar, quantificar, classificar e analisar informações de um todo (KAUARK; MANHÃES; MEDEIROS, 2010) - teve como amostra 144 alunos superdotados de escolas do Irã, país no qual a pesquisa foi desenvolvida. Vale ressaltar que o contexto dos alunos que participaram da pesquisa nesse país, equivale ao ensino fundamental adotado pela conjuntura educacional brasileira. Para tal, foi utilizado alguns questionários para a coleta: Bar-On EQ-i, FFMQ e Questionário de autrregulação (Self-Regulation Questionnaire - SRQ).

Bortoletto e Boruchovitch (2013) desenvolveram um estudo de natureza empírica com 298 estudantes de um curso de Pedagogia de uma instituição pública e uma privada do estado de Minas Gerais/BR, com o objetivo de investigar as relações entre as estratégias de aprendizagem e a regulação emocional desses alunos. Com uma abordagem quantitativa e utilizando-se de análise estatística descritiva e inferencial, as autoras aplicaram os seguintes instrumentos: Escala de Estratégias de Aprendizagem para Estudantes Universitários (EEA-U) e Escala de Estratégias de Regulação Emocional para Adultos.

O estudo de Cruvinel e Boruchovitch (2010) foi caracterizado como uma pesquisa empírica de natureza mista, ou seja, utilizou-se de análises quantitativas e qualitativas. A referida pesquisa teve como objetivo principal descrever os passos da construção de um instrumento qualitativo de modo a investigar as estratégias de regulação emocional de estudantes brasileiros. A amostra foi composta por 54 alunos de $3^{\mathrm{a}}$ e $4^{\mathrm{a}}$ séries do Ensino Fundamental de uma escola pública da cidade de Campinas/SP. Os participantes foram submetidos a uma entrevista e pranchas de avaliação sobre a regulação emocional de alunos do ensino fundamental (EPRE). Este instrumento é composto por 24 questões que visam investigar a percepção, monitoramento e estratégias de regulação emocional das crianças.

Embora diferentes, os estudos selecionados demonstraram grande propriedade na discussão do gerenciamento emocional. Nos estudos teóricos, a literatura, apesar de ainda ser considerada escassa, apresenta a relevância do construto de autorregulação emocional e 
seus derivados, como: regulação emocional, inteligência emocional etc. Os estudos empíricos apresentaram a aplicabilidade do construto principal em diversas faixas etárias e etapas de ensino. Com isso, foi possível aferir a gama de possibilidades e conceitos que o estudo das emoções pode ser desenvolvido e analisado.

Outro fator que merece destaque consiste no "qualis" das revistas, ou seja, a avaliação dada às revistas pela plataforma Sucupira disponibilizada pela CAPES (Coordenação de Aperfeiçoamento de Pessoal de Nível Superior) - órgão responsável pela formação pessoal a nível acadêmico do setor público e privado. O sistema de avaliação é formado por estratos que indicam a qualidade do periódico que pode variar entre: A1 - sendo o mais elevado com pontuação equivalente a 100; A2 - com peso 85; B1 - valendo 70 pontos; B2 - equivalente a 55 pontos; B3 - 40 pontos; B4 - com peso de 25 pontos; B5 - valendo 10 pontos; $\mathrm{C}$ - que corresponde ao peso zero. A avaliação mais recente consiste no quadriênio de 2013 a 2016, e a partir deste parâmetro foi possível identificar o qualis das revistas em que os artigos selecionados foram publicados.

O estudo de Barros, Goes e Pereira (2015) intitulado "Parental self-regulation, emotional regulation and temperament: Implications for intervention" foi submetido e publicado na revista Estudos de Psicologia, a qual tem avaliação A2 na área de Educação, A1 na área de Psicologia e A2 na área interdisciplinar. O artigo de Peña-Sarrionandia, Mikolajczak e Gross (2015) com o título "Integrating emotion regulation and emotional intelligence traditions: ameta-analysis" publicado no periódico Frontiers in Psychology, não obteve registro na plataforma sucupira. De igual modo, o estudo de Heshmati e Ahmadkhanloo (2017) intitulado "Emotional Intelligence, Emotional Self-regulation and Dispositional Mindfulness in High School Gifted Students" submetido na revista Mediterranean Journal of Clinical Psychology - MJCP, não apresentou avaliação na plataforma.

No entanto, o artigo das autoras Bortoletto e Boruchovitch (2013) com o título "Learning Strategies and Emotional Regulation of Pedagogy Students" que fora submetido e publicado na revista Paidéia da Universidade de São Paulo, obteve avaliação A1 tanto na área de Educação, como também na área de Psicologia e Interdisciplinar. O estudo intitulado "Regulação Emocional: A Construção de um Instrumento e Resultados Iniciais" das autoras Cruvinel e Boruchovitch (2010), foi publicado na revista Psicologia em Estudo obtendo nota A2 em Educação, A1 em Psicologia e A2 na área Interdisciplinar. Com isso, apesar de da falta de registro de duas revistas mencionadas, percebe-se a relevância dos estudos analisados, visto a avaliação satisfatória a qual obtiveram na plataforma Sucupira.

\section{Multigrafo COM as PALAVRas-CHAVE.}

A partir do levantamento e caracterização dos estudos selecionados, foi possível desenvolver um multigrafo, que neste estudo consiste na associação entre os principais descritores dos artigos utilizando-se das palavras-chave dos artigos (RAMOS, 2015). O multigrafo, por meio de medidas de centralidade, oferece uma visualização sistematizada da interação entre os construtos analisados a partir do material colhido. O grafo (G) - ou "sociograma" como também é conhecido - consiste em um conjunto de vértices (V) atrelados a um determinado número de arestas (A) que podem ser simétricos e valorados, ou seja, os vértices são os pontos de encontro e as arestas consistem nas linhas que ligam um vértice ao 
outro. Este breve conceito pode ser representado pela expressão " $\mathrm{G}=(\mathrm{V}, \mathrm{A})$ " (FREITAS, 2010; RAMOS, 2015). O objetivo deste tipo de análise consiste em mensurar a interação entres os atores ou objetos da rede por meio de medidas de centralidade.

Para este estudo, foram utilizadas cinco medidas de centralidade: (1) Grau de centralidade In-Degree - identifica os principais atores da rede e o grau de conexões que ele recebe ; (2) Grau de centralidade Out-Degree - também apresenta os principais construtos da rede, porém aponta as conexões que ele realiza a partir dos vértices; (3) Betweenness Centrality - refere-se a centralidade de intermediação, ou seja, mostra a importância de uma variável que está mediando o controle de informações entre os vértices; (4) Closeness Centrality - esta medida informa a distância entre os elementos, ou seja, a quantos "passos" um vértice está do outro; (5) Eigenvector Centrality - responsável por identificar o número de conexões realizadas por um vértice, além de apontar o grau de centralidade dos outros nós aos quais está ligado (RAMOS, 2015; HIGGINS; RIBEIRO, 2018)

$\mathrm{Na}$ Tabela 3 é possível identificar as palavras-chave que apresentaram maior significância dentro da análise estabelecida. A partir do coeficiente de cada medida de centralidade apresentada pode-se perceber a interação e a influência desses construtos. Vale ressaltar que a medida adotada para a construção do Grafo de análise (FIGURA 1) foi a "Eigenvector Centrality", sendo as outras complementares a esta.

A partir da Tabela 3, é possível identificar que os construtos "Emotional intelligence, Emotional competence, Emotion regulation e Coping" apresentaram os mais altos escores na maioria das medidas apresentadas, seguidas de "Review e Meta-analysis" que apresentaram menores escores. Estes construtos foram destacados pelo número de vezes que eles apareceram nos estudos selecionados, apontando para a relevância desses conceitos nas discussões realizadas.

A variável "Emotional intelligence" - traduzida como inteligência emocional - foi conectada à 5 palavras-chave (in-degree $=0$; out-degree $=7$ ) diferentes do multigrafo, quais sejam: Review, Emotional competence, Coping, Emotion regulation, Meta-analysis, Emotional Self-Regulation, Gifted Students. Na medida Betweenness Centrality, a referida variável apresentou um coeficiente de 0,500 , isso significa que a mesma é a que mais está intermediando a conexão entre outras variáveis na rede, ou seja, o estudo do construto Inteligência emocional é fundamental para compreender a relação entre Regulação Emocional e Competência Emocional. Já a medida Closeness Centrality, o coeficiente foi 0,450, apontando para a breve distância entre os vértices. Na principal medida, Eigenvector Centrality, a variável "Emotional intelligence" apresentou o escore de 0,175, isso significa que esta palavra-chave é a mais central na rede e está relacionada às demais variáveis que constituem o multigrafo.

Esse fato pode ser explicado mediante os resultados, que envolvem a investigação conjunta das categorias inteligência emocional $\mathrm{X}$ regulação emocional $\mathrm{X}$ competência emocional, dos autores Heshmati e Ahmadkhanloo (2017) e Peña-Sarrionandia, Mikolajczak e Gross (2015) que demonstraram a eficácia no gerenciamento das emoções quando o indivíduo possui habilidades que evidenciam certa inteligência emocional. Os autores sugerem que aqueles que apresentam um grau elevado de inteligência emocional, conseguem regular suas próprias emoções, mantendo um estado emocional positivo, além de ter mais competência para lidar com emoções negativas. Esse quadro é invertido quando o indivíduo não desenvolve traços dessa inteligência emocional. 
Tabela 3 - Palavras-chave dos artigos sobre a autorregulação emocional na perspectiva social cognitiva, levantados e medidas de centralidade do multigrafo sobre autorregulação emocional, 2019. (Continua)

\begin{tabular}{|c|c|c|c|c|c|c|}
\hline Palavras-chave & Tradução & In-Degree & Out-Degree & $\begin{array}{c}\text { Between- } \\
\text { ness Cen- } \\
\text { trality }\end{array}$ & $\begin{array}{c}\text { Close- } \\
\text { ness } \\
\text { Centra- } \\
\text { lity }\end{array}$ & $\begin{array}{c}\text { Eigen- } \\
\text { vector } \\
\text { Cen- } \\
\text { trality }\end{array}$ \\
\hline $\begin{array}{l}\text { Emotional Self-Regu- } \\
\text { lation }\end{array}$ & $\begin{array}{l}\text { Autorregula- } \\
\text { ção emocio- } \\
\text { nal }\end{array}$ & 2 & 1 & 1,000 & 0,333 & 0,000 \\
\hline Gifted Students & $\begin{array}{l}\text { Alunos super- } \\
\text { dotados }\end{array}$ & 3 & 0 & 1,000 & 0,333 & 0,000 \\
\hline Mindfulness & $\begin{array}{l}\text { Plena menta- } \\
\text { lidade* }\end{array}$ & 0 & 2 & 0,000 & 0,250 & 0,000 \\
\hline Learning & $\begin{array}{l}\text { Aprendiza- } \\
\text { gem }\end{array}$ & 0 & 3 & 0,000 & 0,333 & 0,000 \\
\hline Metacognition & Metacognição & 1 & 2 & 0,000 & 0,333 & 0,000 \\
\hline Self-control & Autocontrole & 2 & 1 & 0,000 & 0,333 & 0,000 \\
\hline Teacher education & $\begin{array}{l}\text { Formação de } \\
\text { professores }\end{array}$ & 3 & 0 & 0,000 & 0,333 & 0,000 \\
\hline $\begin{array}{l}\text { Estratégias de regula- } \\
\text { ção emocional }\end{array}$ & - & 0 & 2 & 0,000 & 0,500 & 0,000 \\
\hline $\begin{array}{l}\text { Instrumento qualita- } \\
\text { tivo }\end{array}$ & - & 1 & 1 & 0,000 & 0,500 & 0,000 \\
\hline Ensino Fundamental & - & 2 & 0 & 0,000 & 0,500 & 0,000 \\
\hline Emotional regulation; & $\begin{array}{l}\text { Regulação } \\
\text { emocional }\end{array}$ & 0 & 3 & 0,000 & 0,333 & 0,000 \\
\hline Parenting & Parental & 1 & 2 & 0,000 & 0,333 & 0,000 \\
\hline Self regulation & $\begin{array}{l}\text { Autorregula- } \\
\text { ção }\end{array}$ & 2 & 1 & 0,000 & 0,333 & 0,000 \\
\hline
\end{tabular}

Nota: As palavras-chave marcadas $\left(^{*}\right)$ foram traduzidas no seu sentido literal para melhor compreensão, apesar de serem expressões utilizadas cotidianamente na sua língua original.

Fonte: Elaborado pelas Autoras (2019).

A segunda variável que apresentou maior escore foi a "Emotional competence" - traduzida como competência emocional - estabelecendo relação com 4 palavras-chave (in-degree = 1; out-degree $=4$ ), a saber: Emotion regulation, Coping, Review, Meta-analysis. Assim como o construto anterior, a Competência emocional obteve um escore de 0,500 na medida Betweenness Centrality, afirmando que esta variável está mediando as informações de outras palavras-chave, como Emotion regulation e Review. Na medida Closeness Centrality o coeficiente foi de 0,200 , atestando que há pouca distância desta variável às outras, ou seja, a referida variável apresenta um papel importante na rede visto que consegue agrupar outras palavras-chave como Coping e Emotion regulation. Na medida Eigenvector Centrality, a variável em questão apresentou escore de 0,175 na mesma proporção da anterior. 
Tabela 3 - Palavras-chave dos artigos sobre a autorregulação emocional na perspectiva social cognitiva, levantados e medidas de centralidade do multigrafo sobre autorregulação emocional, 2019. (Conclusão)

\begin{tabular}{|c|c|c|c|c|c|c|}
\hline Palavras-chave & Tradução & $\begin{array}{c}\text { In-De- } \\
\text { gree }\end{array}$ & $\begin{array}{c}\text { Out- } \\
\text { Degree }\end{array}$ & $\begin{array}{c}\text { Be- } \\
\text { tween- } \\
\text { ness } \\
\text { Cen- } \\
\text { trality }\end{array}$ & $\begin{array}{l}\text { Closeness } \\
\text { Centrality }\end{array}$ & $\begin{array}{c}\text { Eigen- } \\
\text { vector } \\
\text { Centra- } \\
\quad \text { lity }\end{array}$ \\
\hline Temperament & Temperamento & 3 & 0 & 0,000 & 0,333 & 0,000 \\
\hline $\begin{array}{l}\text { Emotional } \\
\text { intelligence }\end{array}$ & $\begin{array}{l}\text { Inteligência } \\
\text { emocional }\end{array}$ & 0 & 7 & 0,500 & 0,450 & 0,175 \\
\hline $\begin{array}{l}\text { Emotional } \\
\text { competence }\end{array}$ & $\begin{array}{l}\text { Competência } \\
\text { emocional }\end{array}$ & 1 & 4 & 0,500 & 0,200 & 0,175 \\
\hline Emotion regulation & $\begin{array}{l}\text { Regulação } \\
\text { emocional }\end{array}$ & 2 & 3 & 0,500 & 0,200 & 0,175 \\
\hline Coping & Lidar* & 3 & 2 & 0,500 & 0,200 & 0,175 \\
\hline Review & Revisão & 4 & 0 & 0,000 & 0,167 & 0,149 \\
\hline Meta-analysis & Metaanálise & 4 & 0 & 0,000 & 0,167 & 0,149 \\
\hline
\end{tabular}

Nota: As palavras-chave marcadas (*) foram traduzidas no seu sentido literal para melhor compreensão, apesar de serem expressões utilizadas cotidianamente na sua língua original.

Fonte: Elaborado pelas Autoras (2019).

A relevância dessa palavra-chave pode ser compreendida no estudo de Peña-Sarrionandia, Mikolajczak e Gross (2015). Os resultados indicaram que os sujeitos que possuem uma alta inteligência emocional - capacidade consciente de identificar, compreender, expressar e regular suas - conseguem moldar suas emoções desde os primeiros momentos percebidos, além de disporem de uma gama de estratégias de gerenciamento maior que os outros indivíduos. Para corroborar com os achados dos autores, destaca-se a pesquisa de Cruvinel e Boruchovitch (2010) que sugerem programas que visem a conscientização das emoções e das estratégias emocionais em crianças - que, com as devidas adaptações, poderia estender-se a adultos - para assim aumentar a sensação de competência emocional delas.

A variável "Emotion regulation" - traduzida com Regulação emocional - obteve influência na rede (in-degree $=2$; out-degree $=3$ ), estabelecendo relação com três variáveis distintas, são elas: Emotional competence, Coping, Review, Meta-analysis. Na medida Betweenness Centrality, a variável em questão apresentou escore de 0,500 , assim como as anteriores, apontando que esta variável está mediando a relação de outras palavras-chave da rede como Review e Meta-analysis. Da mesma maneira, o coeficiente da medida Closeness Centrality foi de 0,200 com o mesmo valor dos construtos supracitados. O quadro se repetiu na medida Eigenvector Centrality, onde a Regulação emocional apresentou escore de 0,175 correspondendo a uma posição central na rede.

Os autores Heshmati e Ahmadkhanloo (2017) ressaltam que a inteligência emocional, a partir de habilidades como solução de problemas e otimismo, exerce grande influência no processo de autorregulação emocional promovendo maior grau de eficácia. A ausência dessa regulação pode trazer malefícios tanto para o próprio indivíduo como para aqueles que o cercam (BARROS; GOES; PEREIRA, 2015). É importante ressaltar que quanto maior 
o engajamento do indivíduo no seu processo de se regular emocionalmente, maior poderá ser a sua competência emocional e sua habilidade em lidar com tarefas cotidianas como, planejamento, monitoramento e desempenho acadêmico, ou seja, estratégias de natureza metacognitiva (BORTOLETTO; BORUCHOVITCH, 2013).

O construto "Coping" - que seria a maneira de lidar com algo ou alguma coisa apresentou associação (in-degree $=3$; out-degree $=2$ ) com duas palavras-chave, a saber: $R e$ view, Meta-analysis. O coeficiente Betweenness Centrality foi igual a 0,500, assim como as anteriores, apontou a função de intermediação entre essas duas variáveis associadas. O escore alcançado na medida Eigenvector Centrality também foi de 0,175 apontando para a centralidade do conceito Coping no multigrafo.

A palavra "Review" - traduzida como "revisão", fazendo referência a revisão de literatura - apresentou menores escores em todas as medidas de centralidade adotadas. $\mathrm{Na}$ medida in-degree o coeficiente alcançado foi igual a 4 , já na medida out-degree não foi encontrado coeficiente, isso indica a direção da conexão entre as variáveis, sendo assim os construtos X e Y se associam à palavra "Review". Na medida Eigenvector Centrality, a palavra Review apresentou o coeficiente igual a 0,149 , atestando baixa centralidade na rede, baixa capacidade de conexão com outros termos.

A variável "Meta-analysis" - ou meta-análise no português - apresentou um quadro similar a palavra-chave anterior. Com um escore igual a 4 na medida in-degree e escore igual a 0 na medida out-degree, apontando que ela não estabeleceu conexão com outras palavras-chave, mas sofreu influência relacional de outros construtos da rede. Na medida Eigenvector Centrality apresentou escore igual a 0,149, apontando para uma baixa influência dessa palavra-chave no multigrafo.

A compreensão da relevância dessas três últimas palavras-chave em destaque pode ser explicada por meio do estudo de Peña-Sarrionandia, Mikolajczak e Gross (2015), o qual obteve grande destaque durante a análise mediante os resultados obtidos pelos autores que conseguiram envolver diversos construtos significantes, como: Coping, Review, Meta-analysis e a relação desses com Emotional intelligence (inteligência emocional). Neste estudo, que fora mencionado, os autores relacionaram o construto da competência emocional como uma habilidade administrada pela inteligência emocional, além de destacarem sua influência no processo de regulação visto que os indivíduos que percebem e identificam suas emoções tentem a pensar na autorregulação com mais facilidade, consequentemente conseguem lidar com estratégias para tal. Os construtos Coping, Review e Meta-analysis estavam relacionados com a Competência emocional, pois participavam do mesmo estudo dos autores citados.

Esses dados ficam mais visíveis quando se visualiza o grafo gerado (FIGURA 1) a partir da métrica Eigenvector Centrality. Vale ressaltar que a pesquisa considerou, inicialmente, todas as palavras-chave contidas nos estudos selecionados. Entretanto, após a aplicação das medidas de centralidade pelo software NodeXL, apenas seis variáveis foram destacadas visto o resultado nas análises efetuadas, principalmente no que tange a medida Eigenvector Centrality (TABELA 4), pois foi a partir dos resultados dessa perspectiva que as outras foram geradas. O grafo ilustrado na Figura 1 foi gerado em consideração apenas a medida Eigenvector Centrality e pode-se identificar o comportamento de cada palavra-chave no multigrafo. 


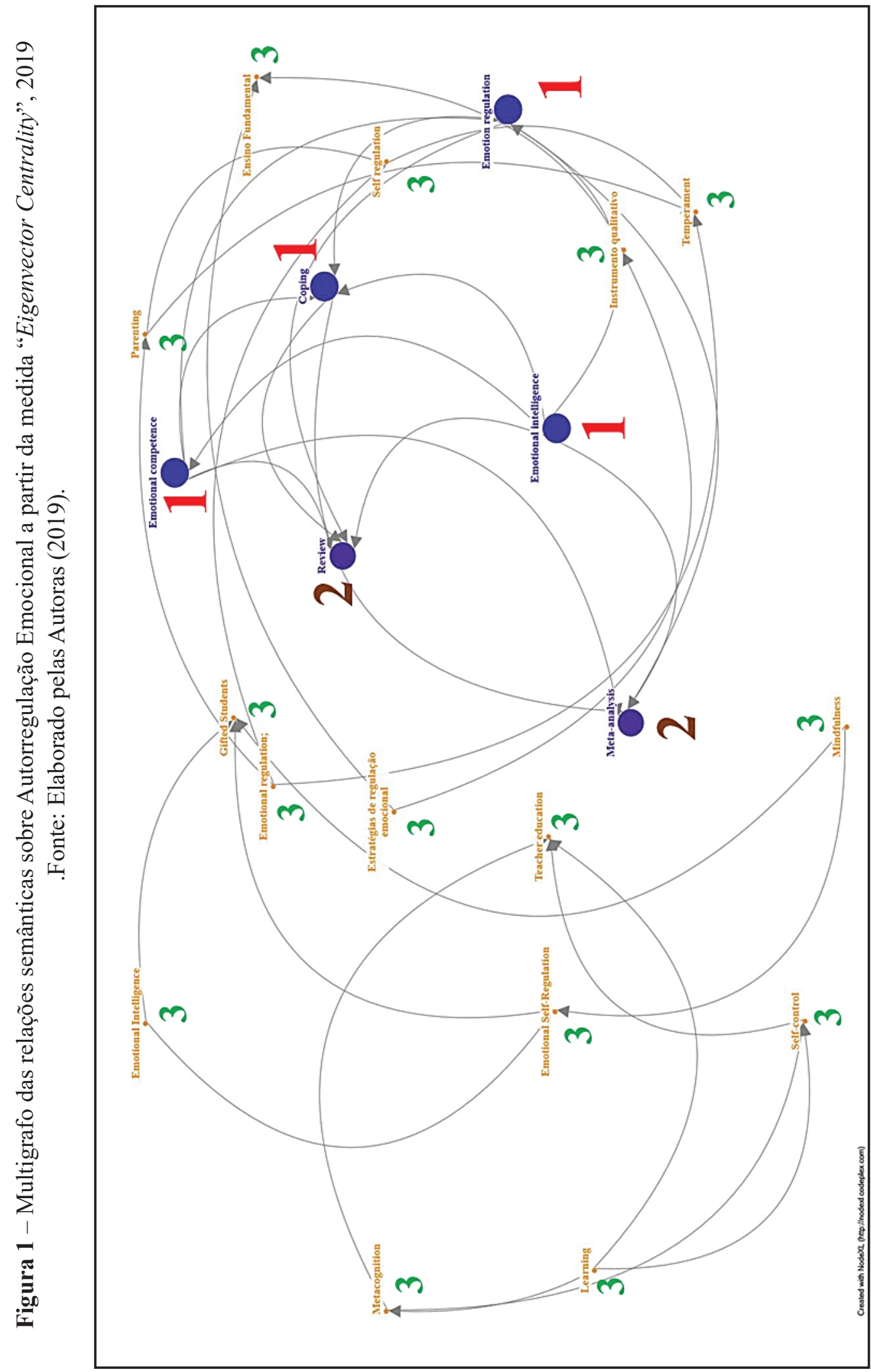


A partir da Figura 1 é possível perceber o conjunto de variáveis que resultaram do levantamento realizado, além das palavras-chave que ganharam destaque por conta dos escores na métrica utilizada: Eigenvector Centrality. Observa-se que na Figura 1 existe uma diferença no tamanho e na cor das esferas do grafo, isso significa que quanto maior e mais azul é o vértice, maior é o número de conexões estabelecidas dessa palavra-chave na rede. Além disso, a orientação das setas demonstra a direção das relações estabelecidas entre os vértices (in-degree - entrada; out-degree - saída).

Mediante essas orientações, percebe-se o destaque de quatro palavras-chave que apresentaram o mesmo peso na rede - com o escore de 0,175 - e estão representadas pelo número “1”, são elas: (1) Emotional intelligence; (2) Emotional competence; (3) Emotion regulation; e (4) Coping. Essas variáveis apresentaram maior capacidade de conexão e influência nos estudos analisados, norteando as discussões dos autores acerca da autorregulação emocional. Marcados com o número "2" estão as variáveis que atestaram escores que indicam baixa capacidade de conexão- igual a 0,149 , portanto acima de zero - que, apesar de menores coeficientes, foram importantes nas discussões dos artigos levantados. As palavras-chave marcadas com o número " 3 " e com cor e tamanhos diferenciados, não apresentaram escores, mas foram citadas na literatura levantada e podem ser evidenciadas em estudos posteriores, indicando ser este um campo ainda insipiente, tendo em vista que estes construtos ainda não são investigados com maior ocorrência por pesquisadores que estudam a autorregulação emocional no recorte temático e temporal analisado nesta revisão.

A partir dos resultados, infere-se a relevância da temática tanto no que tange a estudos teóricos com o objetivo de compartilhar fundamentos sobre aspectos emocionais, como também em estudos empíricos que proporcionam a visibilidade desses aspectos na prática. Entretanto, apesar de sua importância, faz-se necessário um maior desenvolvimento de pesquisas nesta área, principalmente sobre autorregulação emocional.

A ênfase dos estudos no construto da inteligência emocional possibilitou uma maior compreensão sobre a aplicação do gerenciamento das emoções, porém o principal conceito da pesquisa - autorregulação emocional - obteve pouco destaque nos resultados. A ausência de estudos aprofundados sobre a aplicabilidade da autorregulação emocional é lacunar e evidencia a necessidade do aumento de estudos sobre a temática em todos os contextos educacionais, seja na educação básica ou no ambiente acadêmico.

\section{CONSIDERAÇõES FINAIS}

O estudo das emoções faz-se fundamental para compreender suas possíveis implicações no comportamento humano e no desempenho acadêmico dos sujeitos. A autorregulação emocional é basilar, visto sua função de controle e administração das emoções a fim de alcançar uma estabilidade e, por consequência, a promoção do seu bem-estar em diversos contextos inclusive na rotina intensa da pós-graduação.

O objetivo inicial deste artigo consistiu em construir um panorama das pesquisas nacionais e internacionais sobre autorregulação emocional na perspectiva social cognitiva em alunos da pós-graduação por meio de uma revisão integrativa da literatura, delimitando-se ao período de 2008 a 2019. Entretanto, com a escassez de estudos relacionando a temática 
com o contexto da pós-graduação, percebeu-se a necessidade de retirar o descritor "pós-graduação" e ampliar as buscas.

Após a aplicação de todos os critérios e a seleção de cindo estudos, verificou-se a existência de uma rede identificada a partir das palavras principais do estudo de autorregulação emocional comprovando a importância do referido tema, além de sua extensa aplicação nos mais diferentes aspectos da vida humana, especialmente no contexto acadêmico.

Apesar do pequeno número de estudos selecionados, percebeu-se que a autorregulação emocional envolve várias habilidades como a capacidade de identificar e lidar com as emoções tanto negativas quanto positivas, além de estratégias metacognitivas que influenciam diretamente o desempenho do sujeito - planejamento, monitoramento, autoavaliação e regulação.

Outro construto que foi bastante relacionado com a autorregulação emocional foi a inteligência emocional que consiste no gerenciamento efetivo das emoções mantendo emoções positivas como a alegria e modificando emoções negativas a exemplo da raiva, tristeza, medo, dentre outras. Utilizar a inteligência emocional no processo de regulação, tende a dinamizar e efetivar os resultados desse gerenciamento. Além disso, a literatura ainda apontou que a falta da autorregulação emocional pode afetar não só a vida de quem está realizando esse processo, mas pode influenciar o comportamento de outras pessoas que o cercam.

Quanto a metodologia dos estudos selecionados, viu-se que apenas dois artigos foram de natureza teórica e os demais, três, apresentaram métodos empíricos. Ambos são válidos e importantes para a compreensão e conscientização sobre o gerenciamento das emoções.

Apesar da relevância deste estudo, conhece-se algumas limitações como a abertura a outras perspectivas teóricas para além da Teoria Social Cognitiva, além da utilização de outros tipos de materiais como dissertações e teses. Outra limitação identificada consiste na definição dos conceitos de "autorregulação emocional" e "regulação emocional", tal aproximação tende a dificultar a seleção de materiais específicos e pode causar confusão na compreensão desses construtos.

Para novas pesquisas, considera-se as limitações já citadas e pesquisas de natureza empírica tanto de caráter quantitativo quanto qualitativo para que seja possível analisar a autorregulação emocional em outros contextos, como a educação básica, gradação e, especialmente, a pós-graduação.

\section{REFERÊNCIAS}

AZZI, R. G. Introdução à teoria social cognitiva. Série Teoria Social Cognitiva em contexto educativo. São Paulo: Casa do Psicólogo, 2014.

BANDURA, A. Evolução da teoria cognitiva social. Em KG Smith e MA Hitt (Eds.), Grandes mentes na gestão. Oxford: Oxford University Press, p. 9-35, 2005.

BANDURA, A. Social Cognitive Theory of Self-Regulation. Organizational behavior and human decision processes. v. 50, 2 ed, p. 248-287, 1991. 
BANDURA, A. Social cognitive theory: Na agentic perspective. 1999.

BANDURA, A. Social Foundations of Thought and Action: A Cognitive Social Theory. Englewood Cliffs: New Jersey, 1986.

BANDURA, A.; CAPRARA, G. V.; BARBARANELLI, C. Role of affective selfregulatory efficacy in diverse spheres of psychosocial functioning. Child Development, v. 74, n. 3, p. 769-782, 2003.

BANDURA, A.; AZZI, R. G.; POLYDORO, S. Teoria Social Cognitiva: conceitos básicos. Porto Alegre: Artmed, 2008.

BARDIN, L. Análise de conteúdo. Tradução de Luís Antero Reta e Augusto Pinheiro. Lisboa, Portugal: Edições 70, 1977.

BARROS, L.; GOES, A. R.; PEREIRA, A. I. Auto-regulação parental, regulação emocional e temperamento: implicações para a intervenção. Estudos de Psicologia (Campinas), v. 32, p. 295-306, 2015.

BATISTA, J. B. V. et al. Transtornos mentais em professores universitários: estudo em um serviço de perícia médica, 2016.

BORTOLETTO, D.; BORUCHOVITCH, E. Estratégias de Aprendizagem e de Regulação Emocional de Estudantes de Pedagogia. Paidéia (Ribeirão Preto) [online], v.23, n.55, p.235-242, 2013.

BRANDTNER, M; BARDAGI, M. Sintomatologia de depressão e ansiedade em estudantes de uma universidade privada do Rio Grande do Sul. Gerais, Rev. Interinst. Psicol. [online], v.2, n.2, p. 81-91, 2009.

CAPRARA, G.; VECCHIONE, M.; BARBARANELLI, C.; ALESSANDRI, G. Emotional Stability and Affective Self-regulatory. Efficacy Beliefs: Proofs of Integration between Trait Theory and Social Cognitive Theory. European Journal of Personality, 2013.

CARLOTTO, M. S. A síndrome de Burnout e o trabalho docente. Psicol. estud. [online], v.7, n.1, p.21-29, 2002.

CRUVINEL, M.; BORUCHOVITCH, E. Regulación emocional: construcción de un instrumento y resultados preliminares. Psicologia em Estudo, v. 15, n. 3, p. 537-545, 2010.

DAMÁSIO, A. R. O erro de Descartes: emoção, razão e cérebro humano. São Paulo: Companhia de Letras, 1996. 
DEMO, Pedro. Pesquisa: Princípio científico e educativo. São Paulo: Cortez, 4ª ed, 1996.

FRIED, L. J. Professores do ensino sobre regulação da emoção na sala de aula. Jornal Australiano de formação de professores, 2011.

FRIED, L. J. Uso de estratégias de regulação de emoções e motivação na sala de aula do ensino médio. Tese de doutorado. Universidade da Austrália Ocidental, 2010.

GARBER, J.; WALKER, L. S.; ZEMAN, J. Somatization symptoms in a community sample of children and adolescents: Further validation of the Children's Somatization Inventory. Psychological Assessment, v.3, p. 588-595, 1991.

GARNEFSKI, N.; KRAAIJ, V.; SPINHOVEN, P. Negative Life Events, Cognitive Emotion Regulation, and Emotional Problems. Personality and Individual Differences, v. 30, p. 1311-1327, 2001.

GROSS, JAMES J. Emotion regulation: Affective, cognitive, and social consequences. Psychophysiology: Cambridge University Press. Printed in the USA, p. 281-291. 2002

GROSS, JAMES J. Emotion Regulation: Past, Present, Future. Stanford University, California, USA: Cognition and emotion, n. 13, p. 551-573, 1999.

GROSS, JAMES J. The Emerging Field of Emotion Regulation: An Integrative Review. Review of General Psychology. v. 2, n.5, p. 271-299, 1998.

HESHMATI, R.; AHMADKHANLOO, E. Inteligência emocional, autorregulação emocional e mindfulness disposicional em alunos inteligentes do ensino médio. Mediterranean Journal of Clinical Psychology, v. 5, n. 2, 2017.

HIGGINS; S. S.; RIBEIRO, A. C. A. Análise de redes em Ciências Sociais. Brasília: Enap,2018.

KAUARK, F. d. S.; MANHÃES, F. C.; MEDEIROS, Carlos Henrique. Metodologia da pesquisa: um guia prático. Itabuna, BA: Via Litterarum, 2010.

PEÑA-SARRIONANDIAA.; MIKOLAJCZAK M.; GROSS JJ. Integrating emotion regulation and emotional intelligence traditions: a meta-analysis. Psychol. 2015.

POLYDORO, S. A. J. Promoção da autorregulação da aprendizagem: contribuições da Teoria Social Cognitiva. Porto Alegre: Letra, 2017.

RAMOS, M. F. H. Modelo Social Cognitivo de Satisfação no Trabalho e Eficácia Coletiva: Percepções sobre a docência. Belém, 2015. 239 p. Tese. Universidade Federal do Pará, 
Núcleo de Teoria e Pesquisa do Comportamento, Programa de Pós-graduação em Teoria e Pesquisa do Comportamento.

SANTOS, D.; PRIMI, R. Desenvolvimento socioemocional e aprendizado escolar: uma proposta de mensuração para apoiar políticas públicas. São Paulo: Instituto Ayrton Senna, 2014.

SCHUNK, D. H. Learning theories: an educational perspective. $6^{\circ}$ ed. Boston, MA: Pearson, p. $117-163,2012$.

SOUZA, M. T. d.; SILVA, M. D. d.; CARVALHO, R. d. Revisão integrativa: o que é e como fazer. Einstein (São Paulo), v. 8, p. 102-106, 2010.

STORRIE K.; AHERN K; TUCKETT A. A systematic review: Students with mental health problems--a growing problem. Int J Nurs Pract, n. 16, v. 1, p. 1-6, 2010.

WHITTEMORE, R.; KNAFL, K. The integrative review: updated methodology. J Adv Nurs, 2005. Disponível em: < https://pubmed.ncbi.nlm.nih.gov/16268861/>.

\section{DADOS DAS AUTORAS}

Jamille Gabriela Cunha da Silva

Mestre em Educação pela Universidade Federal do Pará (UFPA). jamillegabriela22@, gmail.com

\section{Luciana Amaral Garcia}

Mestre em Educação pela Universidade Federal do Pará (UFPA). luje24504@gmail.com

\section{Maély Ferreira Holanda Ramos}

Pós-Doutora em Teoria e Pesquisa do Comportamento pela Universidade Federal do Pará (UFPA).maelyramos@hotmail.com

Submetido em: 24-06-2020

Aceito em: 31-03-2021 